Application of neutron interferometry to the measurement of thin film density

W. E. Wallace, D. L. Jacobson, M. Arif, and A. loffe

Citation: Appl. Phys. Lett. 74, 469 (1999);

View online: https://doi.org/10.1063/1.123038

View Table of Contents: http://aip.scitation.org/toc/apl/74/3

Published by the American Institute of Physics

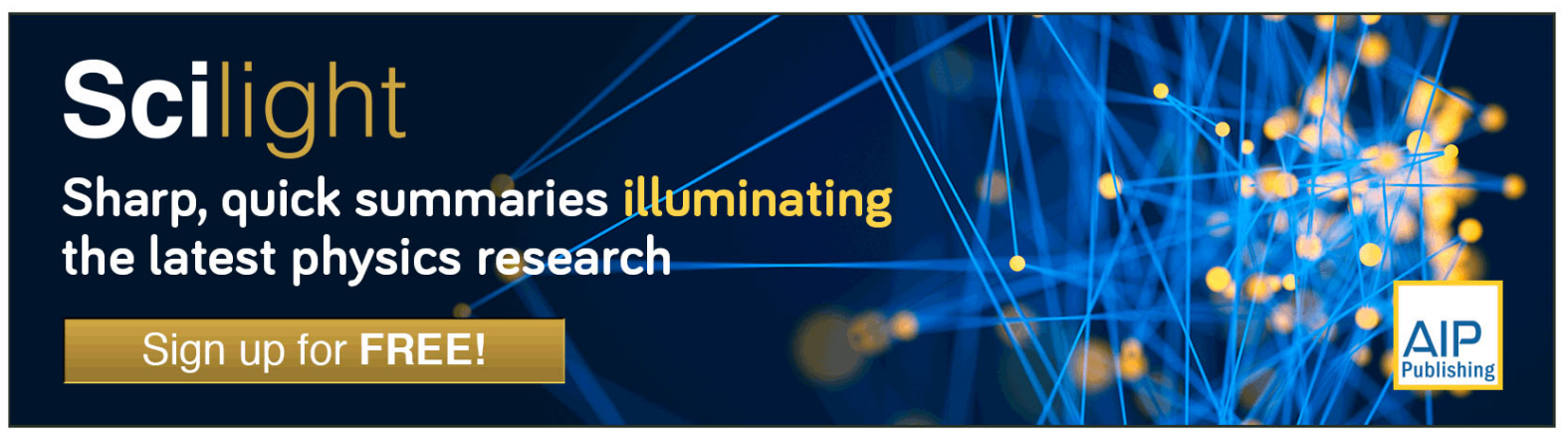




\title{
Application of neutron interferometry to the measurement of thin film density
}

\author{
W. E. Wallace, ${ }^{\text {a) }}$ D. L. Jacobson, M. Arif, and A. loffe ${ }^{\text {b) }}$ \\ National Institute of Standards and Technology, Gaithersburg, Maryland 20899
}

(Received 31 August 1998; accepted for publication 9 November 1998)

\begin{abstract}
The application of neutron interferometry to the measurement of the atom density of polymer thin films $(<1 \mu \mathrm{m}$ thick) supported on silicon substrates is described. Polymer films were chosen primarily for their fixed, well-defined stoichiometry; however, the technique is applicable to films of any elemental composition. The wavelength-independent phase shift of a beam of thermal neutrons passing through the sample gives a measure of the product of the film atom density, the film thickness, the lattice spacing of the silicon interferometer, and the scattering lengths of the constituent elements of the film. The film thickness was found by x-ray reflectivity while the other two parameters are well-defined quantities. The technique does not rely on complex mathematical modeling of physical processes nor on thin film standards for data interpretation. With some refinements, neutron interferometry is envisioned as an important tool in the creation of thin films having well-defined densities which will be useful in the calibration of many analysis techniques.

(C) 1999 American Institute of Physics. [S0003-6951(99)00603-8]
\end{abstract}

Density is a fundamental characteristic of solids. When specifically measured it has been found to be a strong function of synthesis conditions for many materials prepared in thin film form. Some examples include diamond-like carbon, ${ }^{1}$ metals, ${ }^{2}$ silica, ${ }^{3}$ and amorphous silicon. ${ }^{4}$

The methods to measure thin film density can be placed into three categories each having limits to its ultimate accuracy. The first is the direct weighing of a film of known area and thickness. Here uncertainties in the three separate measurements of thickness, area, and total mass are compounded. Alternatively, a property that depends on density is measured, and the film density indirectly inferred. A prominent example is $\mathrm{x}$-ray reflectivity where the critical angle for reflection is related directly to density. ${ }^{5}$ This type of technique requires either calibration standards, or careful mathematical modeling of physical processes (or both), with their attendant difficulties. In the third category are compound methods where the total coverage of material in the thin film, for example measured by Rutherford backscattering spectrometry, is divided by film thickness. These methods depend on accurately measuring the total radiation dose, as well as on knowing the solid angle of detection for the scattered or emitted radiation.

Neutron interferometry ${ }^{6-9}$ falls into the third category with some important differences. The product of the thin film density and the film thickness is calculated from the phase shift of the neutron beam passing through the sample. Measurement of this phase shift is very accurate and neither requires knowledge of the total neutron dose nor of a solid angle for detection. In this way two potential sources of uncertainty are removed. Furthermore, only simple curve fitting (sinusoidal and parabolic) is required, without reliance on thin film standards, making neutron interferometry a good

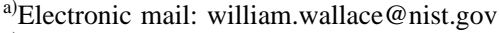

${ }^{b)}$ Also at: Department of Physics and Astronomy, University of Missouri, Columbia, MO 65211.
}

potential candidate for absolute thin film density determination. Combined with an independent measurement of the film thickness, the density of the film can be determined from the neutron phase shift. This is done by applying the following:

$$
\Delta \phi_{s}=-N b \lambda t_{\text {effective }},
$$

where $\Delta \phi_{s}$ is the phase shift, $N$ is the atom density of the sample, $b$ is the coherent neutron scattering length of the thin film material, $\lambda$ is the incident neutron wavelength, and $t_{\text {effective }}$ is the effective sample thickness (i.e., the path length traversed by the neutron beam). When the sample consists of a thin film on a substrate a second measurement is necessary to determine the phase shift from the substrate alone. In most instances the phase shift can be measured within a relative expanded uncertainty of $0.01 \%$ or better. The magnitude of this phase shift is directly dependent on the wavelength and its attendant uncertainty. To eliminate this complication, nondispersive (i.e., wavelength independent) neutron interferometry ${ }^{10,11}$ was employed.

The experiments were performed on a silicon-singlecrystal, three-bladed, Laue-Laue-Laue type interferometer (Fig. 1). The beam enters the interferometer from the top of the diagram where it is split into two coherent beams by Bragg diffraction from (111) silicon lattice planes of the splitter blade. The sample is placed in one arm of this split beam such that the sample surface is parallel to the (111) planes in the interferometer blades, that is, in the nondispersive position. After one beam passes through the sample, the two beams reflect off the (111) planes in the mirror blade and are recombined in the mixer blade after which they reach the detectors creating sinusoidal intensity oscillations as a function of angular position of the phase shifter, $\delta$. In this case the sample thickness is given by $t_{\text {effective }}=t / \sin \theta_{B}$ where $t$ is the true sample thickness and $\theta_{B}$ is the Bragg diffraction angle for the (111) planes of silicon. Substituting $t_{\text {effective }}$ into Eq. (1) along with Bragg's law $\left[\lambda=2 d \sin \theta_{B}\right.$ where $d$ is the (111) interplanar spacing for silicon] yields the following: 


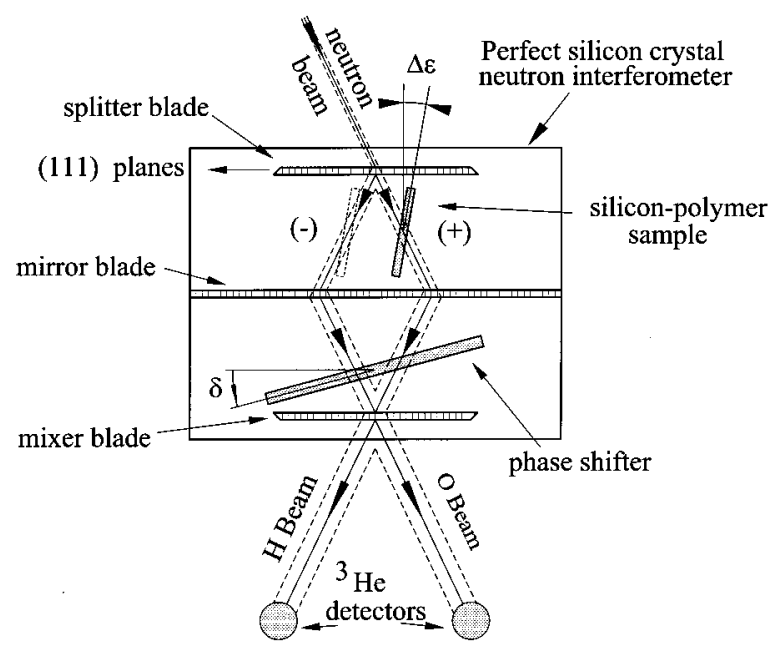

FIG. 1. Diagram of the neutron interferometry experiment. The phase shifter is rotated through the angle $\delta$ to create an interferogram. The sample alignment $\Delta \epsilon$ is adjusted until the difference is phase shift between the sample in the positive position and in the negative position is a minimum.

$$
\Delta \phi_{s}=-2 d N b t .
$$

Thus, the atom density of the sample $N$ depends only on the measured phase shift $\Delta \phi_{s}$, the (111) interplanar spacing of silicon, the coherent neutron scattering length of the thin film material, and the true sample thickness. The phase shift can be very accurately measured. The lattice constant for silicon is a well-known quantity ${ }^{12,13}$ and the scattering lengths of carbon, deuterium, and hydrogen (the constituents of the deuterated polystyrene thin film) are also well known. ${ }^{14}$

The challenge in measuring the phase shift in the nondispersive geometry is the need to place the sample surface parallel to the silicon (111) planes of the interferometer. This angular alignment is termed $\epsilon$, and $\epsilon_{0}$ is the term given to the nondispersive sample position that allows Eq. (2) to be applied with assurance. To insure that this condition is met, the sample is placed in one arm of the interferometer close to the nondispersive position, an interferogram is taken by varying $\delta$, and the phase shift due the sample is measured. Then the sample is translated, without rotation, to the other arm of the interferometer, and a second phase shift, opposite in sign to the first, is measured. When this difference between these two phase shift values, $\Theta$, is a minimum (as shown in Fig. 2) the sample, when placed in either arm of the interferometer, is in the nondispersive position and the following applies:

$$
\Theta=4 d N b t \text {. }
$$

A polymer was chosen to demonstrate this technique because homogeneous thin films, with a well-defined chemical composition, could be prepared by spin coating (from hydrogenated toluene) with subsequent annealing to remove residual solvent $\left(125^{\circ} \mathrm{C}, 2 \mathrm{~h}\right.$, under vacuum). Deuterated polystyrene was used due to the large bound coherent scattering length of the deuterium nucleus which enhanced the phase shift arising from the polymer thin film. The substrates were silicon (100) wafers, approximately $400 \mu \mathrm{m}$ thick, from which the native oxide had been removed using buffered oxide etch.

Figure 2 shows $\Theta$ as a function of $\epsilon$ for a deuterated polystyrene thin film supported on a silicon substrate as well

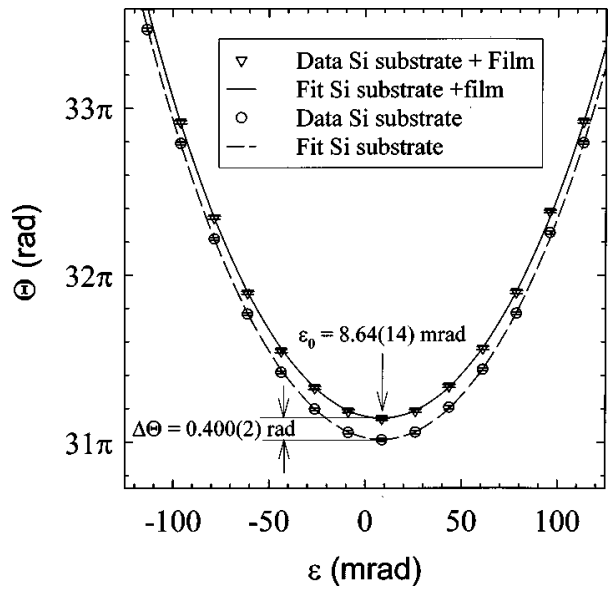

FIG. 2. Neutron phase shift, $\Theta$, vs sample misalignment angle, $\epsilon$, for the polystyrene film supported on a silicon substrate (circles), and for the silicon substrate alone (squares). The solid lines are parabolic fits to the data. The difference in $\Theta$ at the minima give the phase shift due solely to the polystyrene thin film.

as for the silicon substrate alone. It can be shown that for small $\epsilon$ the shape of this curve is parabolic ${ }^{11}$ and is fit as such in Fig. 2. A value of 8.64 (14) mrad was found for $\epsilon_{0}$ which leads to a difference in the phase shift between the film plus substrate, and that of the substrate alone, $\Delta \Theta$, of 0.400 (2) rad. (Throughout the text numbers in parentheses give the combined standard uncertainty.) This value can then be substituted for $\Theta$ into Eq. (3) to determine the density of the thin film.

Figure 3 shows the x-ray reflectivity curve for the particular thin film whose neutron interferometry measurement is given in Fig. 2. The reflectivity was performed with copper $K \alpha_{1}$ radiation $(\lambda=0.1540 \mathrm{~nm})$ having a wavelength spread, $\Delta \lambda / \lambda$, of $1.3 \times 10^{-4}$ and a beam divergence of $5.8 \times 10^{-5} \mathrm{rad}$. The interference fringes apparent in the figure are a sensitive measure of the film thickness. Calculation of the film thickness, ${ }^{15}$ shown by the solid line, gave a value of $521.0(0.6) \mathrm{nm}$ for this film.

Applying Eq. (3) using, in addition to the measured phase shift and measured film thickness, a value of

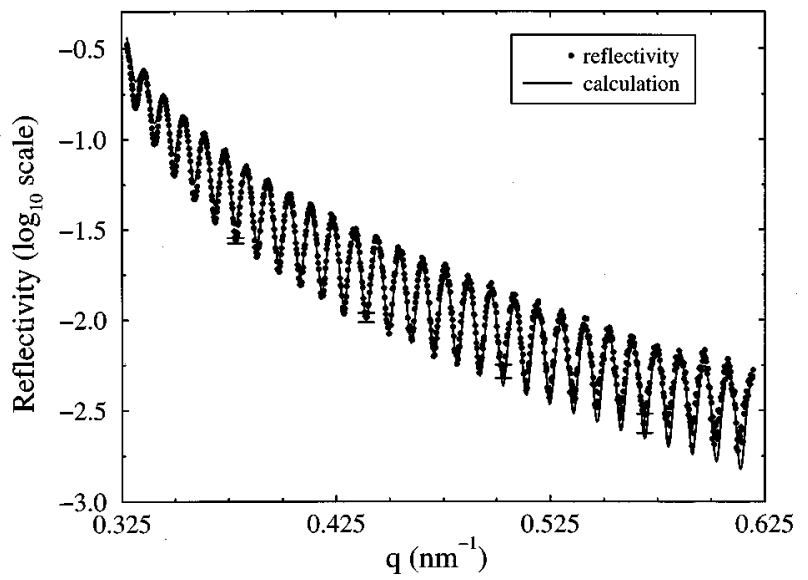

FIG. 3. X-ray reflectivity from the polystyrene thin film supported on its silicon substrate. The $\log _{10}$ of the reflectivity is plotted vs the momentum transfer of the reflected $\mathrm{x}$-ray normal to the sample surface, $q$. The interference fringe spacing is a sensitive measure of the film thickness. The solid line is an exact calculation of the fringe spacing used to determine the film thickness. Some representative uncertainties for the data are given. 
0.31356006 (3) $\mathrm{nm}$ for the silicon (111) spacing, ${ }^{12,13,16}$ the bound coherent neutron scattering lengths of $6.671(4) \times 10^{-13} \mathrm{~cm}$ for deuterium, $-3.7406(11)$ $\times 10^{-13} \mathrm{~cm}$ for hydrogen, and $6.6460(12) \times 10^{-13} \mathrm{~cm}$ for carbon $^{14}$ normalized by the measured film composition ${ }^{17}$ (yielding $6.5544(43) \times 10^{-13} \mathrm{~cm}$ for the polystyrene under study), the atom density of the deuterated polystyrene thin film was found to be $9.339(48) \times 10^{22}$ atoms $/ \mathrm{cm}^{3}(\sim 0.5 \%$ combined standard uncertainty). This value is $3.2 \%$ lower than the bulk density of (hydrogenated) polystyrene. ${ }^{18}$ (The possible change in atom density (not mass density) due to deuteration has not been explicitly considered). Earlier work on the same polystyrene, prepared in the same fashion, using twin neutron reflectivity ${ }^{19}$ found the value for thinner films $(6.5-75.0 \mathrm{~nm})$ to be equal to the bulk value but only to within a few percent due to measurement uncertainty primarily found in the data fitting. Thus, neutron interferometry is envisioned as a way to create more accurate thin film density standards which can be used in the calibration of many types of instruments.

Finally, the accuracy of the measurement could be enhanced by using a substrate made of a zero-phase-shift alloy, that is, an alloy comprised of elements having positive and negative neutron scattering lengths such that the overall scattering length (and, therefore, phase shift) is zero. In this way a separate measurement of the substrate phase shift would not be necessary and thinner films could be measured because uncertainty due to the substrate would be absent.
One of the authors (A.I.) acknowledges support from the National Science Foundation-Physics Division, Grant No. 9603559, and NIST-UMC Cooperative Agreement 8H0008.

${ }^{1}$ Y. Huai, M. Chaker, J. N. Broughton, E. Gat, H. Pepin, T. Gu, X. Bian, and M. Sutton, Appl. Phys. Lett. 65, 830 (1994).

${ }^{2}$ S. M. Heald and B. Nielsen, J. Appl. Phys. 72, 4669 (1992).

${ }^{3}$ H. Yamada, J. Appl. Phys. 82, 4916 (1997).

${ }^{4}$ T. Haage, U. I. Schmidt, H. Fath, P. Hess, B. Schroder, and H. Oechsner, J. Appl. Phys. 76, 4894 (1994).

${ }^{5}$ W. E. Wallace and W. L. Wu, Appl. Phys. Lett. 67, 1203 (1995).

${ }^{6}$ H. Rauch, Science 262, 1384 (1993).

${ }^{7}$ S. A. Werner, Class. Quantum Grav. 11, A207 (1994).

${ }^{8}$ H. Rauch, E. Seidl, A. Zeilinger, W. Bauspiess, and U. Bonse, J. Appl. Phys. 49, 2731 (1978).

${ }^{9}$ S. Tasaki, T. Kawai, and T. Ebisa, J. Appl. Phys. 78, 2398 (1995).

${ }^{10}$ A. Ioffe and M. Vrana, Phys. Lett. A 231, 319 (1997).

${ }^{11}$ A. Ioffe, D. L. Jacobson, M. Arif, M. Vrana, S. A. Werner, P. Fischer, G. L. Greene, and F. Mezei, Phys. Rev. A 58, 1475 (1998).

${ }^{12}$ E. R. Cohen and B. N. Taylor, Rev. Mod. Phys. 59, 1121 (1987).

${ }^{13}$ E. G. Kessler, A. Henins, R. D. Deslattes, L. Nielsen, and M. Arif, J. Res. Natl. Inst. Stand. Technol. 99, 1 (1994).

${ }^{14}$ V. F. Sears, Neutron News 3, 26 (1992).

${ }^{15}$ J. F. Ankner and C. J. Majkrzak, Proc. SPIE 1738, 260 (1992).

${ }^{16}$ The silicon lattice spacing was not corrected for changes in ambient temperature nor pressure away from the published measurement conditions. The expected change is too small to affect the value for the atom density of the polystyrene thin film or its uncertainty.

${ }^{17}$ The composition was found from ion scattering methods to be $C_{1}{ }^{2} H_{0.98}{ }^{1} H_{0.02}$.

${ }^{18}$ T. Ougizawa, G. T. Dee, and D. J. Walsh, Polymer 30, 1675 (1989).

${ }^{19}$ W. E. Wallace, N. C. Beck Tan, W. L. Wu, and S. Satija, J. Chem. Phys. 108, 3798 (1998). 